\title{
Mit einer Stimme sprechen
}

Philip Bruggmann, Julia Kind, Thilo Beck

Arud Zentrum für Suchtmedizin, Zürich

\author{
Der Artikel von Barben et al. zeigt auf, dass der ungeregelte Zugang zu E-Zigaretten \\ aus Präventionssicht unhaltbar ist, insbesondere hinsichtlich Jugendschutz. Wir \\ unterstützen die Forderung nach möglichst konsequenten und kohärenten Mass- \\ nahmen zur Regulierung des Tabakmarkts, denn nur so kann langfristig eine Ab- \\ nahme der Raucherprävalenz erreicht werden. Von suchtmedizinischer Seite ist \\ klar, dass Harm Reduction dabei eine Rolle spielen muss.
}

Der Artikel [1], der auf einer Übersetzung des Positionspapiers der European Respiratory Society beruht, verpasst es leider, den Begriff Harm Reduction in seiner eigentlichen Bedeutung zu verwenden. Zwei suchtmedizinische Ansätze - Harm Reduction und Entwöhnung - werden vermischt; sie erfüllen jedoch jeweils einen unterschiedlichen Zweck und verfolgen ein anderes Ziel. So ist im Artikel wiederholt die Rede von Harm Reduction als «Hilfe zur Raucherentwöhnung». Harm Reduction ist jedoch ein klar definierter Begriff in der Suchtmedizin. Die Verwendung im Artikel von Barben et al. widerspricht der Definition und Bedeutung von Harm Reduction.

Harm Reduction wurde 1995 von Single folgendermassen definiert [2]: «Das Hauptmerkmal der Harm Reduction ist die Akzeptanz der Tatsache, dass von einigen Drogenkonsumenten nicht erwartet werden kann, dass sie ihren Drogenkonsum zum jetzigen Zeitpunkt einstellen. Die Schadensminderung ist neutral gegenüber den langfristigen Zielen der Intervention, während sie den kurzfristigen realisierbaren Zielen eine hohe Priorität einräumt. [...] Der Kern des Konzepts besteht darin, die negativen Folgen des Drogenkonsums zu mildern, während der Drogenkonsum zumindest kurzfristig anhält.»

\section{Abstinenz funktioniert nicht für alle}

In den letzten 20 Jahren hat sich in der Suchtmedizin die Erkenntnis durchgesetzt, dass die Abstinenz nicht das einzige Therapieziel sein darf und dass das dogmatische Festhalten daran vielfach sogar kontraproduktiv ist. Es ist eine Tatsache bei allen Abhängigkeiten von psychoaktiven Substanzen, dass eine Abstinenzerreichung nicht für alle Betroffenen realistisch ist. Dies gilt auch für den Tabakkonsum: Während eine Mehrheit zwar gern mit dem Rauchen aufhören würde, liegen die effektiven Abstinenzraten bei Personen, die medizinische Unterstützung in Anspruch nehmen, je nach angewandter Massnahme im Schnitt bei lediglich 5 bis 15\%. [3]. Leider reicht der häufig vorhandene Wille zur Abstinenz in vielen Fällen nicht aus, um eine Abstinenz zu erreichen. Doch Abhängigkeitserkrankungen sind kein Zeichen von Willensschwäche. Vielmehr handelt es sich bei einer Abhängigkeitserkrankung um ein chronisches Leiden mit bescheidenen Heilungsraten - wenn die Abstinenz als Heilung angesehen wird. Vor diesem Hintergrund muss eine Reduktion von gerauchten Zigaretten und der Umstieg auf alternative Nikotinprodukte als möglicher - wenn auch im Vergleich zum kompletten Verzicht suboptimaler-Behandlungspfad anerkannt werden.

\section{Harm Reduction ist nicht Harm Elimination}

Hier setzt die Harm Reduction an, und hier nehmen auch alternative Nikotinprodukte zur herkömmlichen Zigarette einen Platz ein, auch wenn sie, wie zum Beispiel die E-Zigaretten, selbst ein - wenn auch geringeres-Schadenspotential aufweisen. Harm Reduction ist nicht Harm Elimination. Vielmehr ist das vorrangige und erklärte Ziel bei einer Schadensminderung, die negativen Konsequenzen des Konsums so gering wie möglich zu halten. Damit Raucher jedoch auf E-Zigaretten umsteigen und möglichst vollständig auf herkömmliche Zigaretten verzichten, müssen die Geräte bezüglich Förderleistung optimiert sein und die per E-Zigarette zugeführte Nikotindosis muss derjenigen vor dem Umstieg entsprechen. Die aktuell hohen Zahlen an Dual Usern könnten ein Hinweis auf einen generell zu niedrigen Nikotingehalt in E-Zigaretten sein. Somit müsste in der ärztlichen Begleitung des E-Zigarettenkonsums auf die sachgerechte Anwendung qualitativ hochwertiger und sicherer Geräte und auf eine 
ausreichende Dosis von Nikotin geachtet werden, um effizient den Konsum von herkömmlichen Zigaretten zu minimieren [4]. Eine ausreichende Dosierung und die Gewährleistung einer genügenden Anflutung des Wirkeffekts sind auch in der Opioid-Substitution entscheidend für die Verhinderung von Beikonsum.

\section{Marktregulierung statt Verboten}

Das im Artikel diskutierte Verbot von E-Zigaretten und Liquids ist nicht zielführend. Prohibition als Massnahme gegen Substanzkonsum hat sich noch nie bewährt, wie wir vom jahrzehntelangen und erfolglosen

\section{Prohibition als Massnahme gegen Substanz- konsum hat sich noch nie bewährt, wie wir vom jahrzehntelangen und erfolglosen «War on Drugs» wissen.}

«War on Drugs» wissen. Notwendig ist vielmehr ein gut regulierter Markt für alle Formen des Nikotinkonsums. Spielt sich der Markt für Nikotinprodukte hingegen im illegalen Bereich ab, was im Internetzeitalter nicht zu verhindern wäre, verlieren Prävention und Medizin sowohl die Kontrolle über die erhältlichen Produkte als auch an Einflussmöglichkeiten auf die Konsumierenden.

Die wichtigsten Massnahmen, die zu einem Rückgang des Tabakkonsums führen, liegen in der sorgfältigen Ausgestaltung der Regulierungsinstrumente. Dazu gehören:

PD Dr. med.

Philip Bruggmann

Arud Zentrum für

Suchtmedizin

Schützengasse 31

CH-8001 Zürich

Tel. 0583605000

p.bruggmann[at]arud.ch
- Ein regulierter Zugang zu den Produkten, so dass Jugendliche geschützt werden können.

Ein Tabakproduktegesetz, das diese wesentlichen Bedingungen berücksichtigt, bietet eine wichtige Handhabe zur Prävention und zur Abnahme der Raucherprävalenz. Die Wirksamkeit solcher Massnahmen ist belegt, wie auch Barben et al. in ihrem Artikel ausführen: Während die Raucherraten in Ländern mit schwacher Regulation stagnieren, liegt die Raucherprävalenz in Ländern mit ehemals hohen Raucherraten heute bei 10 bis $12 \%$. Die Prävalenzsenkung wird dabei wohl zu einem grossen Teil über die Senkung von neu beginnenden Rauchern und weniger über dauerhafte Abstinenzerlangung erzielt.

Involvierte Mediziner wie zum Beispiel Pneumologen, Kardiologen und Suchtmediziner tun deshalb gut daran, im politischen und gesellschaftlichen Diskurs mit einer Stimme zu sprechen und gut abgestimmt und gut koordiniert gemeinsame Wege zur Eindämmung der Tabakepidemie und ihrer Folgen aufzuzeigen. Das heisst auch, dass die Fachgebiete vom Wissen und von den Erfahrungen der anderen lernen. Von suchtmedizinischer Seite ist klar, dass Harm Reduction, so wie es im eigentlichen Sinne verstanden wird, dabei eine Rolle spielen muss.

Literatur

1 Barben JSM, Zürcher A, Kaelin R, Schmid T, Stambach D, Hammer J, et al. Harm reduction - keine wirksame Strategie zur Tabakbekämpfung. Schweiz Ärzteztg. 2019;100(31-32):1041-4.

2 Single E. Defining harm reduction. Drug Alcohol Rev. 1995;14(3):287-90.

3 Cornuz J, Jacot Sadowski I, Humair JP. Ärztliche Rauchstoppberatung. Die Dokumentation für die Praxis. Bern: Nationales Rauchstopp-Programm; 2015.

4 Gafner B. Mit mehr Nikotin gegen das Rauchen. Tages-Anzeiger 19.2.2019. 